\title{
Xanthomonas oryzae pv. oryzae Response Regulator TriP Regulates Virulence and Exopolysaccharide Production Via Interacting With c-di-GMP Phosphodiesterase PdeR
}

\author{
Haiyun Li, ${ }^{1,2}$ Dingrong Xue, ${ }^{1}$ Fang Tian, ${ }^{1,+}$ Xiaochen Yuan, ${ }^{3}$ Fenghuan Yang, ${ }^{1}$ Huamin Chen, ${ }^{1}$ \\ William Hutchins, ${ }^{4}$ Ching-Hong Yang, ${ }^{3}$ and Chenyang $\mathrm{He}^{1,+}$ \\ ${ }^{1}$ State Key Laboratory for Biology of Plant Diseases and Insect Pests, Institute of Plant Protection, Chinese Academy \\ of Agricultural Sciences, Beijing 100193, China \\ 2 Jingtang Port Office of Hebei Entry-Exit Inspection and Quarantine Bureau, Tangshan 063611, China \\ ${ }^{3}$ Department of Biological Sciences, University of Wisconsin-Milwaukee, Milwaukee, WI 53211, U.S.A. \\ ${ }^{4}$ Carthage College, Kenosha, WI 53140-1994, U.S.A.
}

Accepted 23 December 2018.

PdeR, a response regulator of the two-component system (TCS) with the cognate histidine kinase PdeK, has been shown to be an active phosphodiesterase (PDE) for intracellular cyclic dimeric guanosine monophosphate (c-di-GMP) turnover and positively regulates the virulence of Xanthomonas oryzae pv. oryzae, the causal pathogen of bacterial blight of rice. To further reveal the key components and pathways involved in the PdeR-mediated c-di-GMP regulation of virulence, 16 PdeRinteracting proteins were identified, using the yeast two-hybrid (Y2H) assay. Among them, PXO_04421 (named as TriP, a putative transcriptional regulator interacting with $\underline{P d e R}$ ) was verified via $\mathrm{Y} 2 \mathrm{H}$ and glutathione- $S$-transferase pull-down assays, and its regulatory functions in bacterial virulence and exopolysaccharide (EPS) production were assessed by biochemical and genetic analysis. The REC domain of TriP specifically interacted with the EAL domain of PdeR. TriP promoted the PDE activity of PdeR to degrade c-di-GMP in the presence of PdeK. In-frame deletion in triP abolished the polar localization of PdeR in the cell. Notably, the $\Delta$ triP mutant showed significantly reduced virulence on susceptible rice leaves and impaired EPS production compared with wild type, whereas the double mutant $\Delta t r i P \Delta p d e R$, like $\Delta p d e R$, caused shorter lesion lengths and produced less EPS than $\Delta$ triP. In addition, cross-complementation showed in trans expression of $p d e R$ in $\Delta$ triP restored its EPS production to near wild-type levels but not vice versa. Taken together, our results suggest

H. Li and D. Xue contributed equally to this work.

${ }^{\dagger}$ Corresponding authors: C. He; hechenyang@ caas.cn and F. Tian; ftian@ippcaas.cn

Funding: This work was supported by the grants from the National Natural Science Foundation of China (31370160, 31671990), National Key Basic Research Program of China (2015CB150600) and the Fundamental Research Funds from Chinese Academy of Agricultural Sciences (1610142017017, Y2015JC20) to C. He, and Beijing Natural Science Foundation (5142017) to F. Tian.

*The $\boldsymbol{e}$-Xtra logo stands for "electronic extra" and indicates that three supplementary figures and two supplementary tables are published online.

The author(s) declare no conflict of interest.

๑) 2019 The American Phytopathological Society that TriP is a novel regulator that is epistatic to PdeR in positively regulating virulence expression in $X$. oryzae pv. oryzae.

Two-component systems (TCSs) consisting of histidine kinases (HKs) and response regulators (RRs) are predominant for signal transduction in bacteria (Jung et al. 2012; Stock et al. 2000; Zschiedrich et al. 2016). TCSs help bacteria to sense, respond, and adapt to changes in their surroundings, including environmental and host conditions. Usually, the signal transduction involves autophosphorylation at the histidine residue of a HK upon sensing certain signals and, then, transfer of the phosphoryl group to a conserved aspartate residue on the receiver domain of its cognate RR. Although the majority of RRs function as transcriptional regulators that trigger responses by directly affecting gene expression, there are other types of signaling outputs related to enzymatic regulation or proteinprotein interactions (Gao and Stock 2009; Jung et al. 2012; Skerker et al. 2005).

Bacterial second messengers are intracellular signaling molecules that trigger physiological changes. Cyclic dimeric guanosine monophosphate (c-di-GMP), a nucleotide-type second messenger, has been shown to regulate the bacterium life-style transition from single planktonic cells to a sessile, multicellular biofilm (Povolotsky and Hengge 2012; Römling et al. 2013; Simm et al. 2004). It has also been implicated in regulating other cellular behaviors such as cell-cycle progression, flagellar motility, and virulence (Abel et al. 2011; Fang and Gomelsky 2010; Paul et al. 2010; Yang et al. 2012; Yuan et al. 2015). Extensive structural and functional studies have revealed that synthesis and degradation of c-di-GMP is performed by GGDEF domain-containing diguanylate cyclases (DGCs) and EAL- or HD-GYP domain-containing phosphodiesterases (PDEs), respectively (Schirmer and Jenal 2009). c-di-GMP effectors, which are capable of binding to c-di-GMP, can sense intracellular c-di-GMP levels and trigger physiological changes accordingly (Sondermann et al. 2012). So far, several different types of c-di-GMP effectors have been identified, including PilZ-domains, various transcriptional factors, degenerated GGDEF/EAL domains, riboswitches, and recently defined ATPases of secretion systems (Jones et al. 2015; Roelofs et al. 2015; Sondermann et al. 2012).

Xanthomonas oryzae pv. oryzae causes bacterial leaf blight of rice, which is the most severe bacterial disease of this staple 
crop worldwide. X. oryzae pv. oryzae enters rice leaves through wounds or water pores, leading to systemic infection in the xylem tissue of susceptible rice cultivars (Niño-Liu et al. 2006). Whether or not the disease occurs is largely dependent on the interactions between the type III effectors and their target genes in the host (White and Yang 2009; Zhang and Wang 2013). Besides the type III secretion system, virulence factors such as exopolysaccharides (EPS), extracellular enzymes, and the type II secretion system are also critical for the pathogenesis of $X$. oryzae pv. oryzae (Ray et al. 2000; Sun et al. 2005; Zhou et al. 2013).

In the past few years, we have uncovered several components in the c-di-GMP signaling pathway of $X$. oryzae pv. oryzae. PdeR is an active PDE and a TCS RR and, together with its cognate HK PdeK, positively regulates the EPS production and virulence of $X$. oryzae pv. oryzae (Yang et al. 2012). Similar to PdeR, EdpX1 functions as a PDE to degrade c-di-GMP and promotes EPS production, biofilm formation, and virulence (Xue et al. 2018). A putative DGC, GdpX1, controls EPS production and virulence in a manner opposite to PdeR (Yang

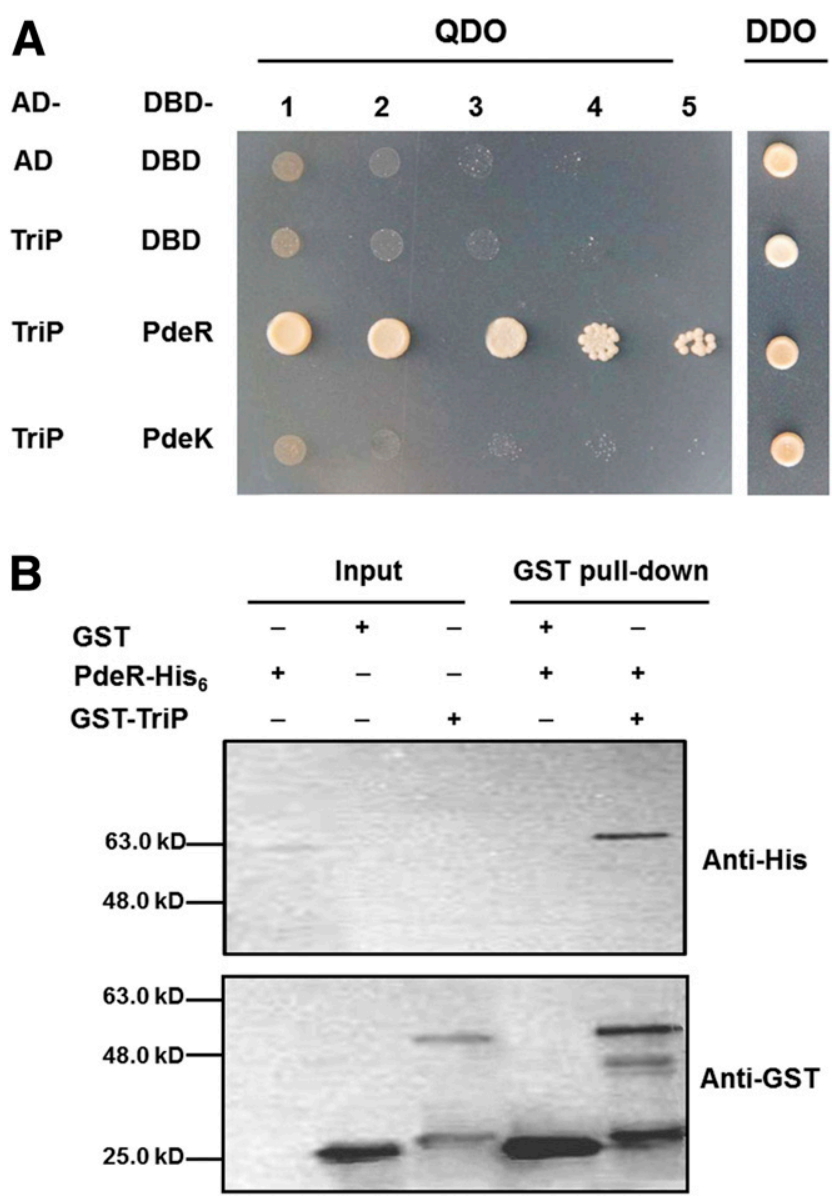

Fig. 1. Interaction between PdeR and TriP was confirmed by yeast twohybrid (Y2H) assay and glutathione-S-transferase (GST) pull-down assay. A, Interaction between full-length PdeR and TriP was detected in the $\mathrm{Y} 2 \mathrm{H}$ assay. The Y2HGold yeast strains carrying pGBKPdeR and Y187 yeast strains carrying pGADTriP were mated and selected on double drop-out (DDO) medium and were further examined on quadruple drop-out (QDO) medium. Serial dilutions (10-fold) were performed to evaluate the interaction strength. Pictures were taken after the cells grew for 2 days. B, Interaction between PdeR and TriP was confirmed by the GST pull-down assay. Purified PdeR-His ${ }_{6}$ fusion protein was incubated with an equal amount of purified GST-TriP and were precipitated by the GST binding beads. The coeluted proteins were detected by Western blotting using antiGST (lower panel) or anti-His (upper panel) antibodies. et al. 2016). This regulation scheme is consistent with the general theory that low c-di-GMP promotes virulence, while high c-di-GMP inhibits virulence. In addition, several different types of c-di-GMP receptors and effectors have been identified from $X$. oryzae pv. oryzae, including the degenerated GGDEF/EAL domain protein Filp, the PilZ domain proteins PXO_00049, PXO_02374, and PXO_02715, and the transcriptional regulator $\mathrm{Clp}$ ( $\mathrm{Li}$ et al. 2013; Yang et al. 2014, 2015).

In this study, we focused on a complicated PdeR-dependent signaling pathway that regulates a plethora of virulence factors in $X$. oryzae pv. oryzae. We identified PXO_04421 as an interactor of PdeR, which was named as TriP (a putative transcriptional regulator interacting with $\underline{P d e R})$. Their genetic and biochemical relationship was also investigated, and the results showed TriP might be epistatic to PdeR in regulating virulence-related traits of $X$. oryzae pv. oryzae.

\section{RESULTS}

\section{The response regulator TriP specifically interacts} with the phosphodiesterase PdeR.

To uncover the PdeR-mediated c-di-GMP signaling pathway and its regulation of virulence in $X$. oryzae pv. oryzae, we started by looking for proteins that interact with PdeR in $X$. oryzae pv. oryzae. Yeast two-hybrid $(\mathrm{Y} 2 \mathrm{H})$ assays were performed using PdeR as a bait expressed in the Y2HGold strain against the prey library of $X$. oryzae pv. oryzae constructed in the host strain Y187. A total of 16 putative PdeR-interacting proteins were found after the screening process (Supplementary Table S1). Among them, PXO_04421 was annotated as an OmpR-family TCS response regulator. The full length of PXO_04421 consisted of an N terminal REC domain (6 to 117 aa) and a $\mathrm{C}$ terminal helix-turn-helix (HTH) DNA-binding domain (157 to 232 aa). Thus, we named it TriP. To test whether TriP is a bona fide interactor of PdeR, we cloned the full length of the triP gene into prey vector pGADT7 and transformed it into the Y187 strain. Interaction of AD-TriP with DBD-PdeR or DBD-PdeK was directly tested, using Y2H assays (Fig. 1A). Yeast zygotes containing AD-TriP and DBD-PdeR showed robust growth on quadruple drop out (QDO) plates in 2 days, suggesting their interaction was strong. In contrast, no interaction between TriP and PdeK was observed. In addition, glutathione- $S$-transferase (GST) pull-down assays were carried out to further confirm the interaction between PdeR and TriP. GST-TriP, GST tag only, and PdeR-His 6 were expressed and purified. Different combinations of the purified proteins were mixed and were then precipitated with GST binding beads. The coeluted proteins were detected by Western blotting, using antiGST or anti-His antibodies. PdeR-His ${ }_{6}$ was the only protein detected when GST-TriP was present in the mixture (Fig. 1B), indicating that TriP could pull PdeR down. Moreover, no interaction between TriP and PdeK was observed (Fig. 1A). These results provide evidence that TriP physically and specifically interacts with PdeR.

\section{The TriP REC domain specifically interacts with the PdeR EAL domain.}

Since both PdeR and TriP are multidomain proteins consisting of the GGDEF-EAL-REC and REC-HTH domains, respectively, we questioned which domains are essential for their interaction. Therefore, $\mathrm{Y} 2 \mathrm{H}$ assays were employed to determine the interaction between structural variations of proteins PdeR and TriP. The N-terminal half of TriP (TriP-N) and its Cterminal half (TriP-C) were cloned into the pGADT7 vectors, and their interactions with DBD-PdeR were tested. The results showed that TriP-N but not TriP-C was able to interact with the 
full-length PdeR (Fig. 2A). Then, we examined which domain of PdeR was essential for its interaction with TriP-N. Each domain of PdeR, including GGDEF(G), EAL(E), and REC(R), were cloned into the pGBKT7 vector. However, we were not able to get the Y2HGold transformants for pGBKT7 carrying PdeR-E (pGBKPdeR-E). Therefore, the interaction between PdeR-E and TriP-N was tested after switching the vectors for these two domains. The results showed that the EAL domain of PdeR specifically interacted with TriP-N (Fig. 2A). These observations suggest that the REC domain of TriP and the EAL domain of PdeR are essential for the two-protein interaction (Fig. 2B).

\section{TriP enhances the PDE activity of PdeR for c-di-GMP turnover.}

Since PdeR harbors PDE activity to degrade c-di-GMP and TriP is identified as a novel interactor of PdeR, it is important to know whether TriP affects the enzymatic activity of PdeR. To test this, colorimetric assays were carried out to examine PDE activities in different protein combinations. GST tag alone or recombinant proteins GST-TriP, PdeR-His 6 , and PdeK-His 6 were purified to homogeneity (Fig. 3A). As expected, PdeR alone did not show any PDE activity. With the addition of ATP and its cognate HK PdeK, the PDE activity of PdeR was significantly increased (Fig. 3B). TriP, which does not harbor any PDE-encoding domain, did not exhibit any c-di-GMP degrading ability. As shown in Figure 3, TriP itself was able to trigger PdeR PDE activity in the absence of PdeK, although the activity was relatively lower. PDE activity of PdeR was enhanced after addition of TriP into the ATP+PdeK+PdeR mixture in a concentration-dependent manner (Fig. 3B), which was an unexpected observation. This experimental data demonstrates that TriP promotes the PDE activity of PdeR in the presence of PdeK.

To provide additional evidence that intracellular c-di-GMP levels are affected by TriP, the c-di-GMP contents in wild type, $\Delta$ triP, $\Delta$ triP(pBTriP), $\Delta p d e R, \Delta p d e K$, and $\Delta t r i P \Delta p d e R$ were examined by liquid chromatography-tandem mass spectrometry (LC-MS/MS) analysis. Like $\Delta p d e R$ and $\Delta p d e K, \Delta t r i P$ showed significant increases in c-di-GMP concentration compared with wild type (Fig. 4). In addition, the elevated c-di-GMP amount was restored to the wild-type level in complementary strain $\triangle t r i P(\mathrm{pBTriP})$. In addition, $\triangle$ triP $\triangle p d e R$ displayed a higher c-di-GMP level than $\Delta$ triP. These findings demonstrate that TriP provides a significant negative influence over intracellular levels of c-di-GMP.

\section{TriP affects polar localization of PdeR}

in X. oryzae pv. oryzae cells.

Subcellular compartmentation of c-di-GMP signaling components has been suggested to facilitate temporal and spatial regulation of specific activities (Güvener and Harwood 2007; Merritt et al. 2010; Paul et al. 2008). PdeR has been demonstrated to physically interact with both its cognate HK PdeK (Yang et al. 2012) and a transcriptional regulator, TriP, in this study. We will next address whether these proteins have specific localization patterns and whether colocalization occurs between them. To test this, C-terminal green fluorescent protein (GFP) fusions of TriP, PdeR, and PdeK were generated and were observed for their subcellular localization in $X$. oryzae pv. oryzae under fluorescence microscopy. As shown in Figure 5, PdeR demonstrated bipolar localization in most cells. Comparatively, PdeK was not only localized to the poles but, also, had multiple foci in most cells. It is possible that PdeK might function as a putative $\mathrm{HK}$ to sense environmental signals. When both PdeK and PdeR are localized to the poles, it might help to increase the rate of phosphorylation from PdeK to PdeR, thus increasing the concurrent PDE activity of PdeR. Different from PdeR and PdeK, which showed specific foci, the signal of TriPGFP diffused throughout the whole cell. However, it is still possible that transient interaction between TriP and PdeR occurs in the cytoplasm.

Since both PdeK and TriP seem to be epistatic to PdeR in the regulatory pathway, we next asked whether PdeK or TriP influences the subcellular localization of PdeR in X. oryzae pv. oryzae. We examined the localization patterns of PdeR-GFP in the $\Delta p d e K$ and $\Delta$ triP mutants (Fig. 5). Interestingly, PdeR retained its bipolar localization in $\triangle p d e K$, the same as the wildtype strain, indicating no effect of PdeK on PdeR localization. However, the bipolar localization pattern disappeared in $\Delta t r i P$, suggesting TriP significantly influenced the subcellular localization of PdeR. These results obviously indicate the necessity of TriP but not PdeK for bipolar localization of PdeR in $X$. oryzae pv. oryzae.

\section{TriP is required for optimal virulence} of $X$. oryzae pv. oryzae in rice.

The observation that TriP influences the PDE activity of PdeR prompted us to examine whether TriP also regulates the virulence of $X$. oryzae pv. oryzae. An in-frame deletion mutant

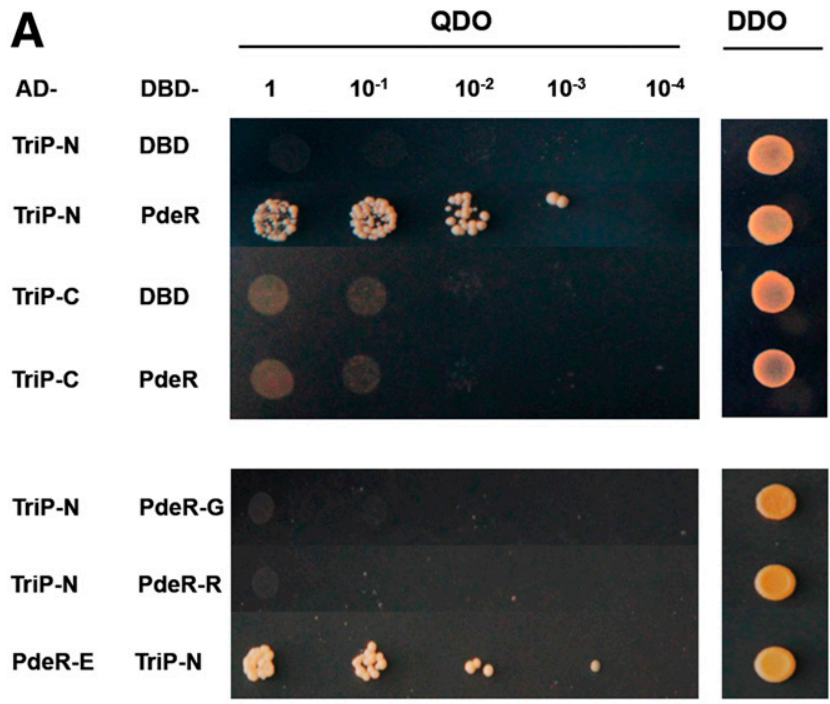

B

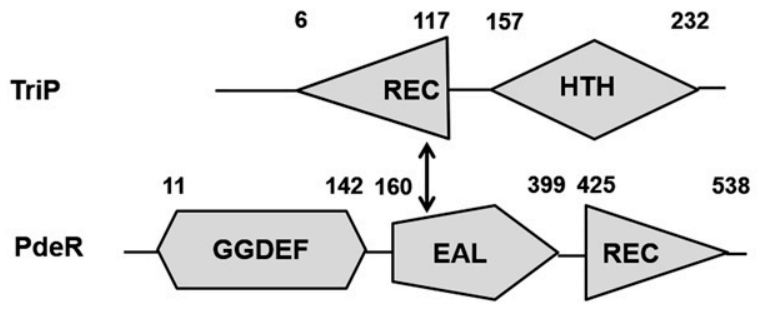

Fig. 2. Determination of specific domains required for the interaction between PdeR and TriP. A, Interactions between different fragments of PdeR and TriP were tested in yeast two-hybrid ( $\mathrm{Y} 2 \mathrm{H})$ assays. Different combinations were detected between Y187 yeast strains carrying pGADT7derived constructs to express the $\mathrm{N}$ terminus of TriP (TriP-N), the $\mathrm{C}$ terminus of TriP (TriP-C), and the EAL domain of PdeR (PdeR-E). Y2HGold yeast strains carrying pGBKT7-derived constructs were used to express PdeR, the GGDEF domain of PdeR (PdeR-G), the REC domain of PdeR (PdeR-R) and TriP-N, respectively. B, Interaction between different domains are schematically summarized. The REC domain of TriP and the EAL domain of PdeR were required for the interaction. Numbers represent amino acid residues where the predicted domains start and end, based on the National Center for Biotechnology Information conserved domain database and the SMART database. 
of triP was tested for its virulence on a susceptible rice variety (Oryza sativa L cv. Nipponbare), using the leaf-clipping method. The disease symptoms and the lesion lengths were scored 14 days postinoculation. Compared with the wild-type strain, disease severity caused by the $\Delta t r i P$ mutant was decreased, resulting in shorter lesion lengths, and the complemented strain containing a plasmid to express full-length triP in trans could restore the diseased phenotypes to nearwild-type levels (Fig. 6). To further characterize the genetic relationship between TriP and PdeR, we constructed a $\triangle$ triP $\triangle p d e R$ double mutant and tested its virulence. The $\triangle t r i P \triangle p d e R$ double mutant caused similar disease symptoms and lesion lengths to the $\Delta p d e R$ mutant, which was less severe than the $\Delta$ triP mutant (Fig. 6). Moreover, to clarify whether the reduction in virulence of the mutants is due to reduction in growth, growth rates of the wild type, $\Delta t r i P, \Delta p d e R$, and $\Delta$ triP $\Delta p d e R$ strains were measured in M210 liquid medium. No significant differences were observed in growth rates between the four strains (Supplementary Fig. S1), suggesting that gene deletion of either triP, pdeR, or both does not significantly affect the viability of $X$. oryzae pv. oryzae under normal growth conditions. Accordingly, these findings reveal that TriP and PdeR are both required for the virulence of $X$. oryzae pv. oryzae to host plants and TriP might act upstream of PdeR in the regulatory pathway of virulence in $X$. oryzae pv. oryzae.

\section{TriP is epistatic to PdeR in regulating EPS production.}

Since EPS is generally regarded as an important virulence factor for $X$. oryzae pv. oryzae and reduced virulence on rice is displayed in $\triangle t r i P$, we tested whether its EPS production was affected. An approximately 10\% decrease in EPS secretion of $\triangle$ triP was observed, compared with that of wild type, while the complemented strain restored EPS production to near wild-type levels (Fig. 7). In contrast to $\triangle t r i P$, a $25 \%$ reduction of EPS was observed in $\triangle p d e R$. In addition, EPS production was examined in the $\triangle t r i P \triangle p d e R$ double mutant, and interestingly, it was reduced to a level that was very similar to that of $\Delta p d e R$. This was consistent with the virulence phenotypes observed previously (Fig. 6). To further investigate their genetic relationship, we constructed cross-complementation strains $\Delta p d e R(\mathrm{pBTriP})$ and
$\Delta$ triP(pBPdeR $)$ and examined their EPS production levels. EPS secreted from $\Delta t r i P(\mathrm{pBPdeR}-\mathrm{GFP})$ was restored to wild-type level, while that from $\Delta p d e R(\mathrm{pBTriP})$ could not be restored (Fig. 7). These observations reveal that TriP might be epistatic to PdeR in positively regulating EPS production of $X$. oryzae pv. oryzae.

\section{DISCUSSION}

Resolving the highly complex c-di-GMP signaling pathway is of utmost importance for a comprehensive understanding of molecular mechanisms underlying virulence expression and

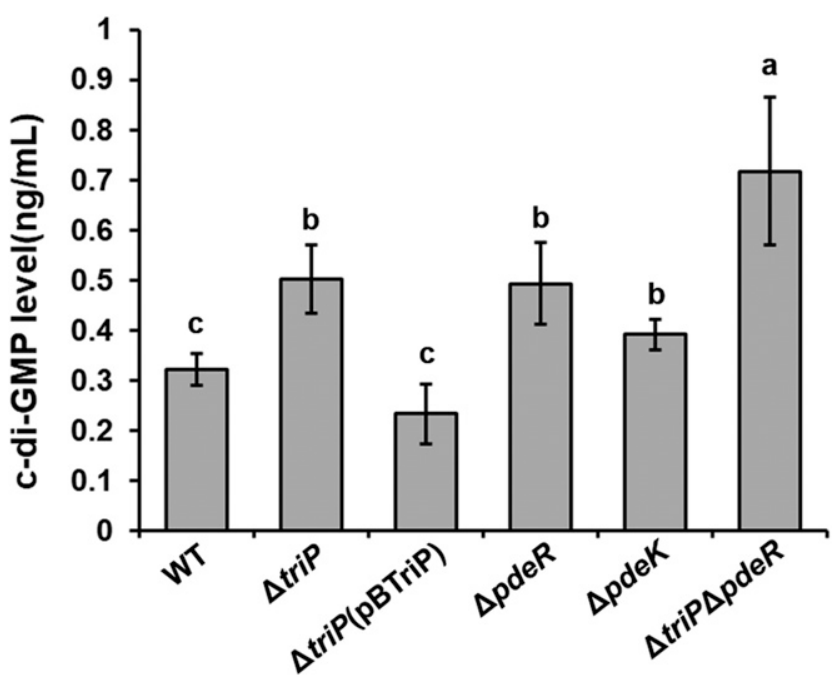

Fig. 4. Measurement of intracellular cyclic dimeric guanosine monophosphate (c-di-GMP) levels of Xanthomonas oryzae pv. oryzae strains. The intracellular c-di-GMP concentrations in wild-type PXO99 ${ }^{\mathrm{A}}, \Delta t r i P$, $\Delta t r i P(\mathrm{pBTriP}), \Delta p d e R, \Delta p d e K$, and $\Delta t r i P \Delta p d e R$ were assayed by liquid chromatography-tandem mass spectrometry. Error bars represent standard deviation from three biological repeats. Lower case letters $(a, b$, and $c)$ indicate significant difference $(P<0.05)$ by Fisher's least significant difference test.
A

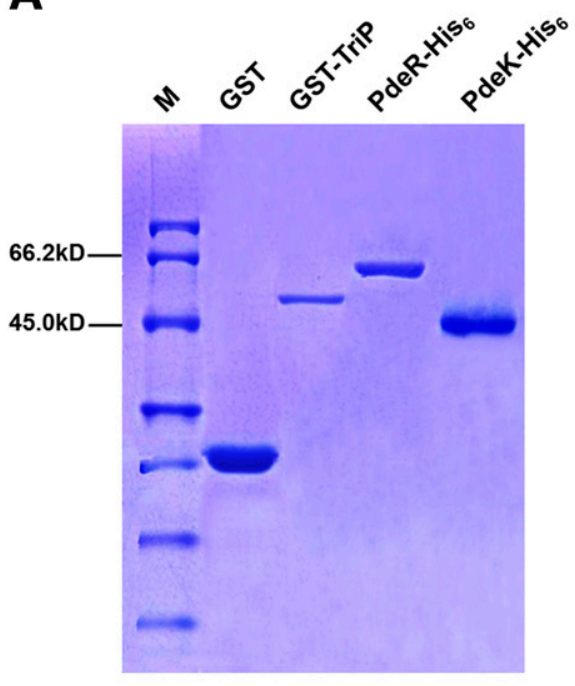

B

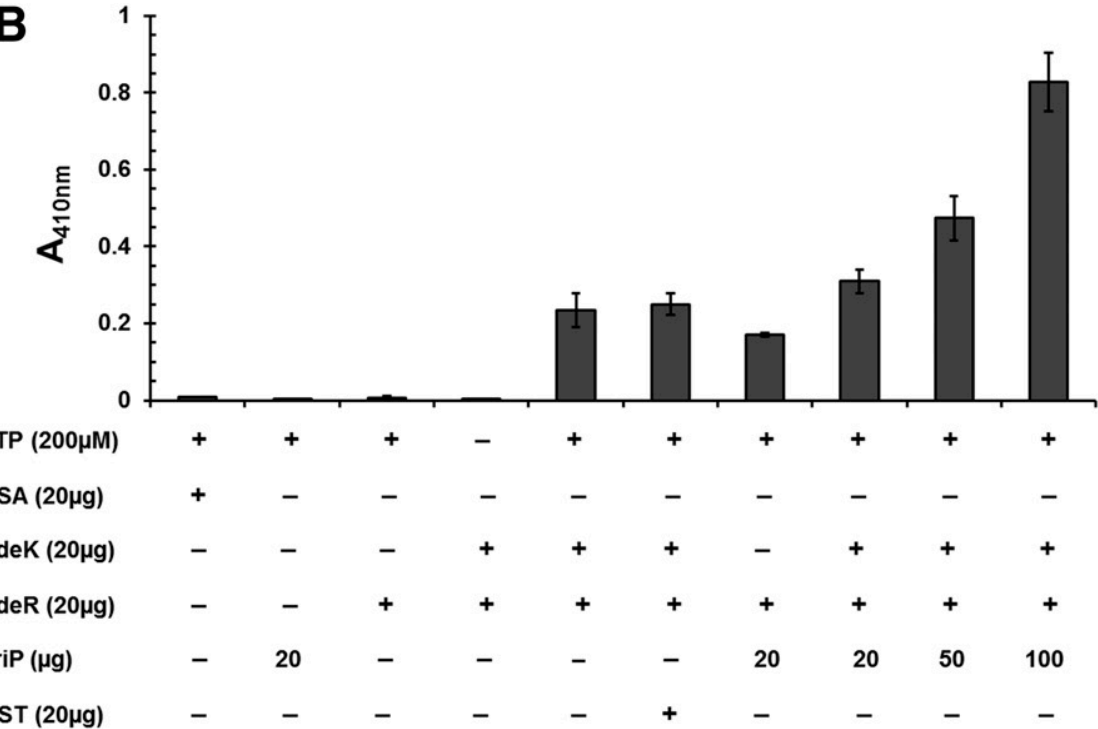

Fig. 3. Colorimetric assay to detect phosphodiesterase (PDE) activity of PdeR. A, The purified proteins of glutathione-S-transferase (GST), GST-TriP, PdeRHis $_{6}$, and PdeK-His 6 were analyzed by sodium dodecyl sulfate polyacrylamide gel electrophoresis. B, Bis (p-nitrophenyl) phosphate substrate was used for the PDE colorimetric reaction, and the hydrolysis of the substrate was detected spectrophotometrically at a wavelength of 410 nm. The error bars represent standard deviations in triplicate. 
regulation in pathogenic bacteria (Römling et al. 2013). We have previously demonstrated that PdeR, an active PDE for cdi-GMP turnover, positively regulates virulence expression in X. oryzae pv. oryzae (Yang et al. 2012). To further reveal key components and pathways of PdeR-mediated c-di-GMP signaling involved in regulation of virulence, we identified and characterized TriP, a novel RR that interacted with PdeR in this study. TriP was demonstrated to enhance PDE activity for c-diGMP hydrolysis and proper intercellular bipolar localization of PdeR. In addition, TriP was shown to positively influence bacterial virulence on rice as well as EPS production. To our knowledge, this is the first study that shows that TriP functions as a novel key regulator of virulence expression via a PdeRmediated c-di-GMP signaling pathway.

The identification of the TriP-PdeR regulatory cascade prompts us to speculate about possible interaction patterns of two TCS RRs in X. oryzae pv. oryzae. It has been generally recognized that $\mathrm{HKs}$ detect and translate input information and RRs control the output during the TCS-mediated signal transduction pathway (Jung et al. 2012; Zschiedrich et al. 2016). In RRs, REC domains typically function as phosphorylation-mediated switches, catalyze their own phosphorylation and dephosphorylation, and activate the output domains, such as HTH DNA-binding or EAL and GGDEF domains (Bourret 2010; Gao and Stock 2009). Based on bioinformatic analysis of TriP that possessed both REC and HTH domains (Fig. 2B), we defined TriP as a 'characteristic' RR. TriP was shown to interact directly with TriK, an OmpR family TCS sensor kinase (Supplementary Fig. S2), indicating TriK/TriP might be a novel pair of TCS in X. oryzae pv. oryzae. More interestingly, PdeR, consisting of the GGDEF-EAL-REC domains, forms a cognate pair with the HK PdeK to regulate bacterial virulence (Yang et al. 2012). Here, we revealed that the TriP REC domain had a novel and additional function in specific binding to the PdeR EAL domain (Fig. 2A), while TriP didn't interact with PdeK (Fig. 1A). We also believe that this is the first report to identify the REC-EAL domain interaction of two RRs, while also illustrating the function of TriP as a PDE interactor in $X$. oryzae pv. oryzae.

Since degradation of c-di-GMP is catalyzed by PDEs, regulation of PDE hydrolytic activity is crucial for modulation of cellular functions (Römling et al. 2017). It has been shown that

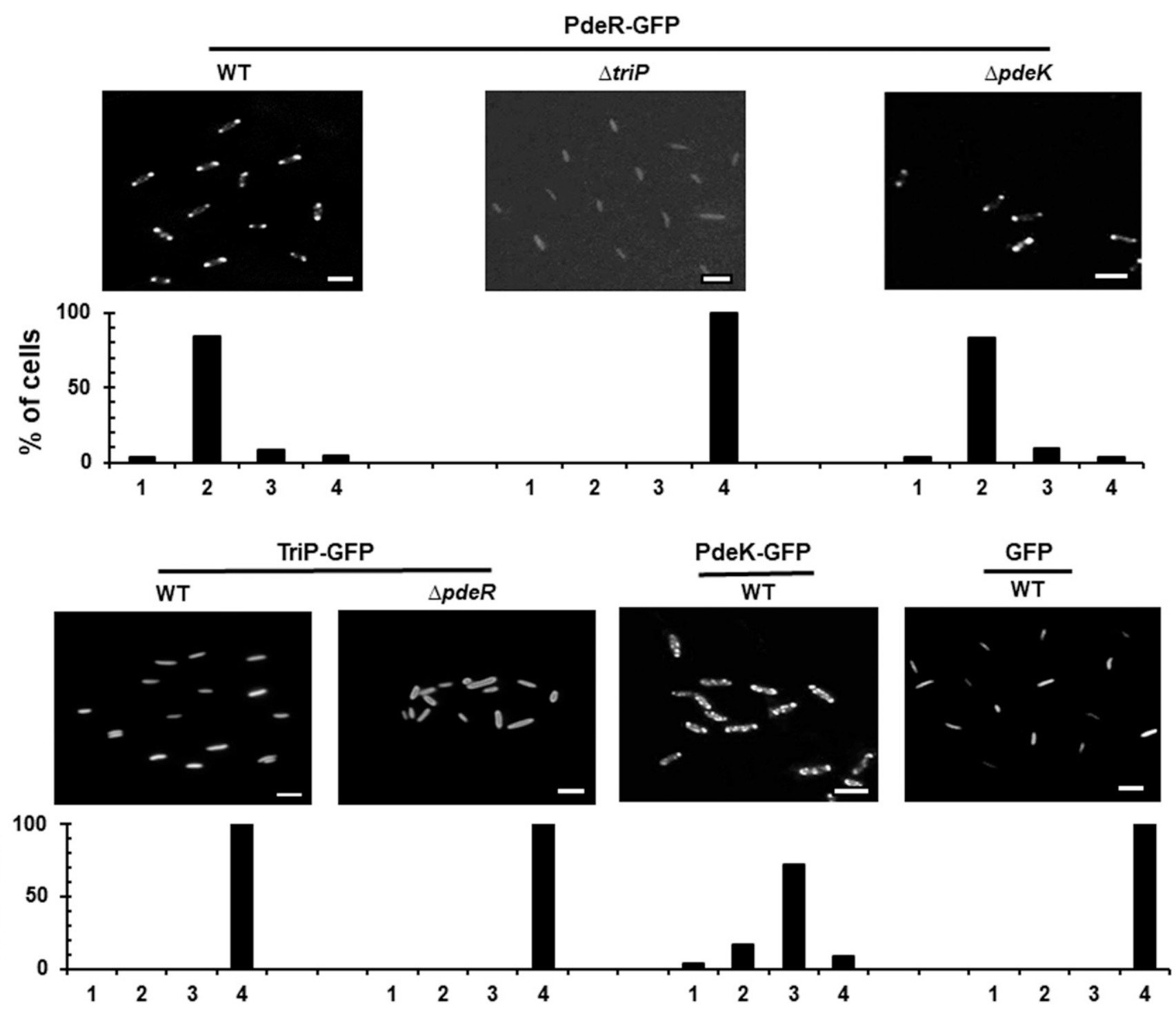

Fig. 5. Subcellular localization of green fluorescent protein (GFP) fusion proteins in Xanthomonas oryzae pv. oryzae strains. X. oryzae pv. oryzae strains were cultured in M210 medium at $28^{\circ} \mathrm{C}$, until the optical density at $600 \mathrm{~nm}=0.6$, were diluted and $3 \mu \mathrm{l}$ of cell suspension was spotted on a glass slide coated with $1 \%$ agarose. Photographs of GFP fusion proteins were taken using a fluorescence microscope (Olympus BX61), and subcellular localization was counted in at least 10 fields of view. The scale bar represents $2 \mu \mathrm{M}$ in length. Numbers indicate different locations: 1, unipolar; 2, bipolar; 3, multisite; and 4, cytoplasmic. 
local c-di-GMP signaling relies on direct protein-protein interactions of one or both a PDE and a DGC with a specific cdi-GMP-binding (Dahlstrom et al. 2015; Lindenberg et al. 2013). Such a protein interaction can establish a local 'source' and 'sink' of c-di-GMP in the immediate vicinity of a c-diGMP-controlled machinery (Hengge 2016). Though more remains to be elucidated regarding the relevant regulatory interaction of TriP with PdeR, the plausible mechanism underlying the physical domain interaction of the two RRs can be postulated. We provide four possible hypotheses: i) allosteric regulation of PdeR may occur upon interacting with TriP in the presence of the substrate c-di-GMP, thereby leading to enhanced enzyme activity of PdeR; ii) TriP regulation of

A
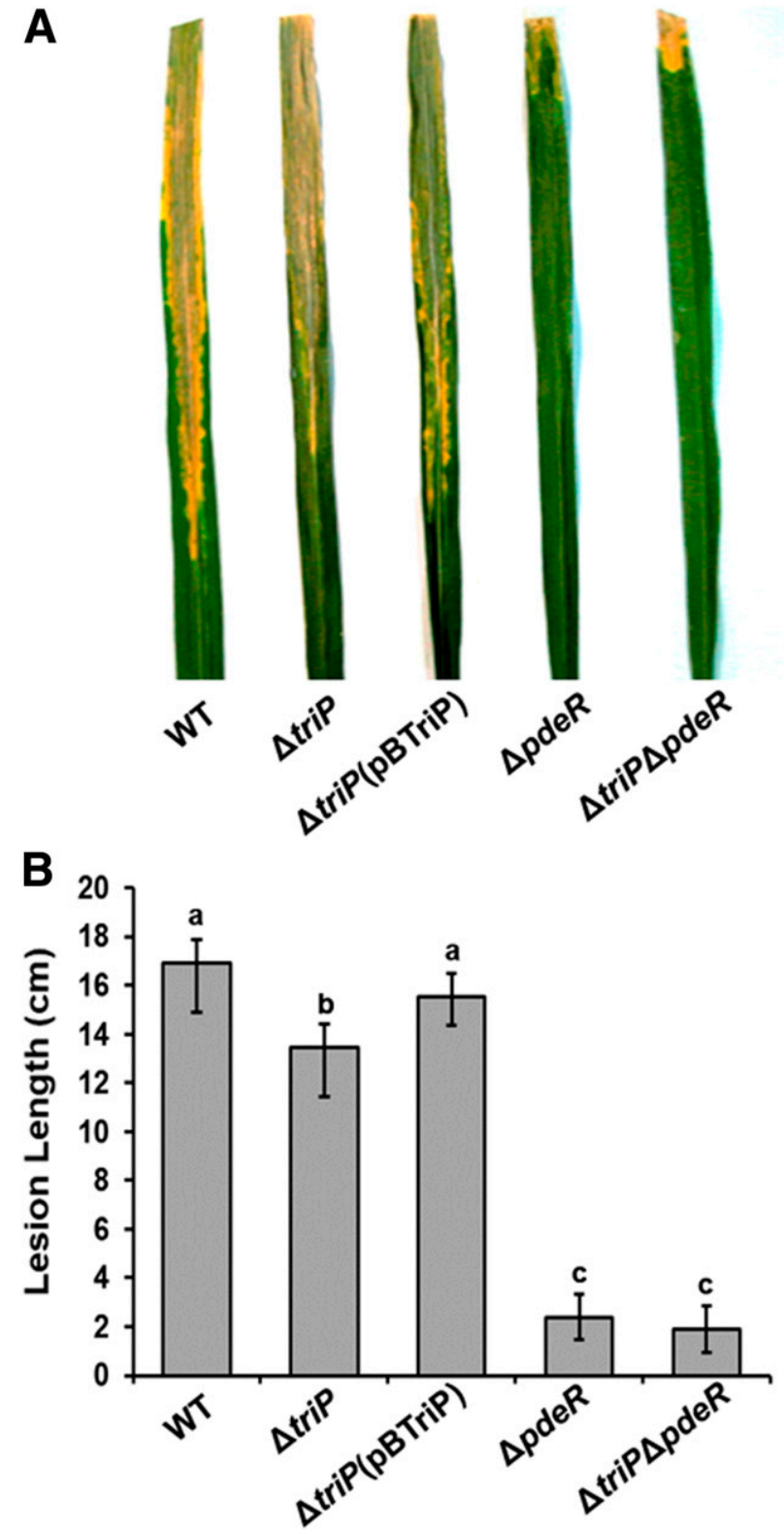

Fig. 6. Virulence test of Xanthomonas oryzae pv. oryzae strains on susceptible rice variety (Oryzae sativa L. cv. Nipponbare). Virulence of $X$. oryzae pv. oryzae strains was assessed by the leaf-clipping method. A, Symptoms were recorded by photography and $\mathbf{B}$, the lesion lengths were measured for individual inoculated leaves 14 days after inoculation. Values are means and error bars represent standard deviations from 20 inoculated leaves in one experiment. The experiment was repeated four times with similar results. Lower case letters $(\mathrm{a}, \mathrm{b}$, and $\mathrm{c}$ ) indicate significant difference $(P<0.05)$ by Fisher's least significant difference test. bipolar localization of PdeR may be at the level of proteinprotein interaction but not transcription, since TriP did not affect the transcription of either $p d e R$ or $p d e K$, as revealed by our unpublished RNA-seq analysis data; iii) the phenomenon that TriP deletion results in significant increases of intracellular cdi-GMP might be due to an influence on the function of TriP itself as a putative response regulator; and iv) TriP itself can activate PdeR PDE activity without PdeK and no direct interaction exists between TriP and PdeK, suggesting TriP might not act as an intermediary between PdeK and PdeR or as a phosphor donor itself. PdeR might be activated independently by both PdeK phosphorylation and TriP interaction. Preliminary bioinformatic analysis of the TriP-PdeR interaction predicted the REC and EAL domain interface structures (Supplementary Fig. S3). To further understand the exact molecular basis underlying this regulatory cascade, providing the crystal structure defining the protein-protein interaction of TriP and PdeR may provide some insights.

We found that TriP was necessary for full virulence in rice and EPS production, one of the most critical pathogenicity factors in $X$. oryzae pv. oryzae. $\Delta$ triP was shown to significantly reduce virulence on rice and impaired EPS production compared with the wild type, whereas the double mutant $\Delta t r i P \Delta p d e R$, like $\Delta p d e R$, caused shorter bacterial blight lesion lengths and produced less EPS than $\Delta$ triP (Figs. 6 and 7). In trans complementation of $p d e R$ in $\Delta$ triP restored its virulence and EPS production to near wild-type levels but not vice versa (Fig. 7). Combined with our previous report that both $\Delta p d e K$ and $\Delta p d e R$ showed decreased virulence on rice but the virulence of $\Delta p d e R$ was more significantly affected (Yang et al. 2012), we believe that $\Delta$ triP was less affected in virulence than $\Delta p d e R$. Thus, we conclude that TriP functions upstream of PdeR in the TriP-PdeR regulatory cascade in $X$. oryzae pv. oryzae.

The impact of TriP on bacterial virulence and EPS production was less than that of PdeR alone, as discussed above; however, $\Delta$ triP showed intracellular c-di-GMP levels similar to

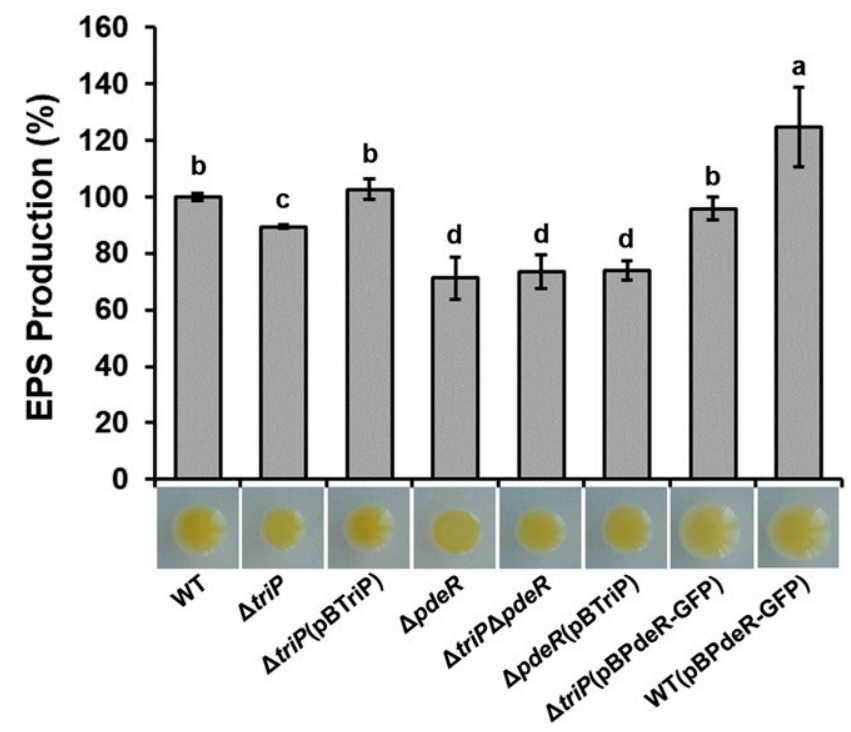

Fig. 7. Quantitative and phenotypic assays for exopolysaccharide (EPS) production of Xanthomonas oryzae pv. oryzae strains. EPS quantitative assays of $\Delta$ triP, $\Delta$ triP(pBTriP), $\Delta p d e R, \Delta t r i P \Delta p d e R, \Delta p d e R(\mathrm{pBTriP}), \Delta$ triP (pBPdeR-GFP), and PXO99 ${ }^{\mathrm{A}}$ (pBPdeR-GFP) strains were determined by the weight method, compared with the level of wild-type PXO99 ${ }^{\mathrm{A}}$. Phenotypes were recorded by a Canon 550D digital camera. Values are means and error bars represent standard deviation from three repeats. Lower case letters $(\mathrm{a}, \mathrm{b}$, and $\mathrm{c})$ indicate significant difference $(P<0.05)$ by Fisher's least significant difference test. 
that of $\Delta p d e R$ (Fig. 4). We speculate that TriP may function in the regulation of bacterial virulence phenotypes on many levels. For example, TriP regulates virulence and EPS production by interacting with PdeR at the protein-protein level, as revealed in this work. A working model outlining TriP regulation of virulence expression via interacting with PdeR in $X$. oryzae pv. oryzae has been proposed (Fig. 8). In addition, TriP may also mediate its impact on virulence expression at the transcriptional level. TriP, a putative transcriptional regulator, may target a set of genes encoding the downstream key components in c-di-GMP signaling pathways, thereby modulating virulence expression in the pathogen. Further RNA-seq analysis of TriP will identify these important components and their roles in the TriP-mediated regulation of virulence in $X$. oryzae $\mathrm{pv}$. oryzae.

Taken together, the identification of the novel regulator TriP will increase our current understanding of the interplay and functionality of RRs, which are involved in the c-di-GMP signaling network in pathogenic bacteria.

\section{MATERIALS AND METHODS}

\section{Bacterial strains, plasmids, and primers.}

Escherichia coli strains were grown at $37^{\circ} \mathrm{C}$ in Luria Bertani (LB) medium (Hanahan 1983). X. oryzae pv. oryzae wild-type strain PXO99 ${ }^{\mathrm{A}}$ (Hopkins et al. 1992) and derived mutant strains were cultured at $28^{\circ} \mathrm{C}$ in $\mathrm{M} 210$ medium $(0.8 \%$ casein enzymatic hydrolysate, $0.5 \%$ sucrose, $0.4 \%$ yeast extract, $0.03 \%$ $\left.\mathrm{MgSO}_{4} \cdot 7 \mathrm{H}_{2} \mathrm{O}, \mathrm{pH} 7.0\right)$ or peptone sucrose agar (PSA) $(1 \%$ peptone, $1 \%$ sucrose, $1 \%$ glutamic acid, $1.5 \%$ agar, $\mathrm{pH} 7.0$ ) medium with relevant antibiotics. The antibiotics were used at the following concentrations: ampicillin, $100 \mu \mathrm{g} / \mathrm{ml}$; kanamycin, $50 \mu \mathrm{g} / \mathrm{ml}$; gentamycin $50 \mu \mathrm{g} / \mathrm{ml}$; and cephalexin $20 \mu \mathrm{g} / \mathrm{ml}$. Bacterial strains, plasmids, and primers used in this study are listed in Table 1.

RNA isolation and cDNA library construction.

To extract RNA from PXO99 ${ }^{\mathrm{A}}$, bacterial strains were grown in $\mathrm{M} 210$ at $28^{\circ} \mathrm{C}$ till it reached an optical density at $600 \mathrm{~nm}$ $\left(\mathrm{OD}_{600}\right)$ of 0.6 to 0.8. A bacterial RNAout (Tiangen Biotech Co., Beijing) kit was used for total RNA isolation according to the manufacturer instructions. Total RNA was treated with DNase I (Tiangen Biotech Co.) to remove DNA contamination. The cDNA library for $\mathrm{Y} 2 \mathrm{H}$ screening was generated using the Make Your Own "Mate \& Plate" Library System (Clontech Laboratories, Mountain View, CA, U.S.A.) according to the manual description. In brief, $5 \mu \mathrm{g}$ of total RNA served as the template for cDNA synthesis utilizing the SMARTIII technology. Double-stranded cDNA less than 400 bp were discarded, using Chroma Spin TE-400 columns, followed by cotransformation with pGADT7 into competent yeast strain Y187. Then, pelleted cells were resuspended in $15 \mathrm{ml}$ of $0.9 \%$ (wt/vol) $\mathrm{NaCl}$ solution and spread on selection agar plates depleting leucine (SD/-Leu). Transformants were pooled and kept in 1-ml aliquots and were stored at $-80^{\circ} \mathrm{C}$ until use.

\section{Y2H screening and assay.}

For $\mathrm{Y} 2 \mathrm{H}$ screening, a 1-ml library aliquot of $X$. oryzae $\mathrm{pv}$. oryzae cDNA library was used as prey, and Y2HGold containing pGBKPdeR was used as the bait strain (Yang et al. 2012). They were mixed and incubated at $30^{\circ} \mathrm{C}$ for $24 \mathrm{~h}$. The mixture was centrifuged and the resuspended pellets were spread onto stringency SD/-Leu/-Trp plates containing aureobasidin $\mathrm{A}$ and $\mathrm{X}$-á-gal (double drop out [DDO/X/A]), followed by higher stringency SD/-Ade/-His/-Leu/-Trp supplemented with aureobasidin $\mathrm{A}$ and $\mathrm{X}$ - $\alpha$-gal $(\mathrm{QDO} / \mathrm{X} / \mathrm{A})$. Total surviving blue colonies on QDO were subjected to polymerase chain reaction $(\mathrm{PCR})$ and sequencing. Sequences were compared with the GenBank database using BLASTn.

For the Y2H assay, the DNA fragments of triP, triP-N, triP$C$, pdeR-G, pdeR-E, and $p d e R-R$ were amplified using primer pairs ADTriPF/R, ADTriP-NF/R, BKTriP-NF/R, ADTriP$\mathrm{CF} / \mathrm{R}$, BKPdeR-GF/R, ADPdeR-EF/R, and BKPdeR-RF/R, respectively. PCR products were cloned into pMD18-T for sequencing. The fragments were then digested from pMD18T-derived constructs and were subcloned into prey vector pGADT7 or bait vector pGBKT7, resulting in pGADTriP, pGADTriP-N, pGADTriP-C, pGADPdeR-E, pGBKPdeR-R, pGBKPdeR-G, pGBKPdeR-E, and pGBKTriP-N (Table 1). The constructs were transformed into the Saccharomyces cerevisiae Y187 and Y2HGold strains, respectively, and were confirmed by PCR. Pairwise yeast mating between haploid cells, each containing pGADT7 and pGBKT7 constructs, was performed in yeast potato dextrose agar at $28^{\circ} \mathrm{C}$ for $24 \mathrm{~h}$, then, the diploid cells were selected on DDO plates (SD/-Leu/-Trp) and were further screened on QDO/X/A plates

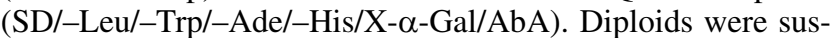
pended in sterile water and four 10-fold serial dilutions were placed on the QDO plates. Growth of the diploids on QDO plates for 2 days indicated strong interactions between the test proteins.

\section{Protein expression and purification.}

The full length of triP was amplified by primer pair GEXTriPF/R, was ligated into expression vector pGEX-6P-1, and was transformed into the E. coli BL21 strain for GSTtagged protein expression. In addition, full-length pdeR and $p d e K$ was cloned into expression vector pET28a for expression as $\mathrm{His}_{6}$-tagged fusions (Yang et al. 2012).

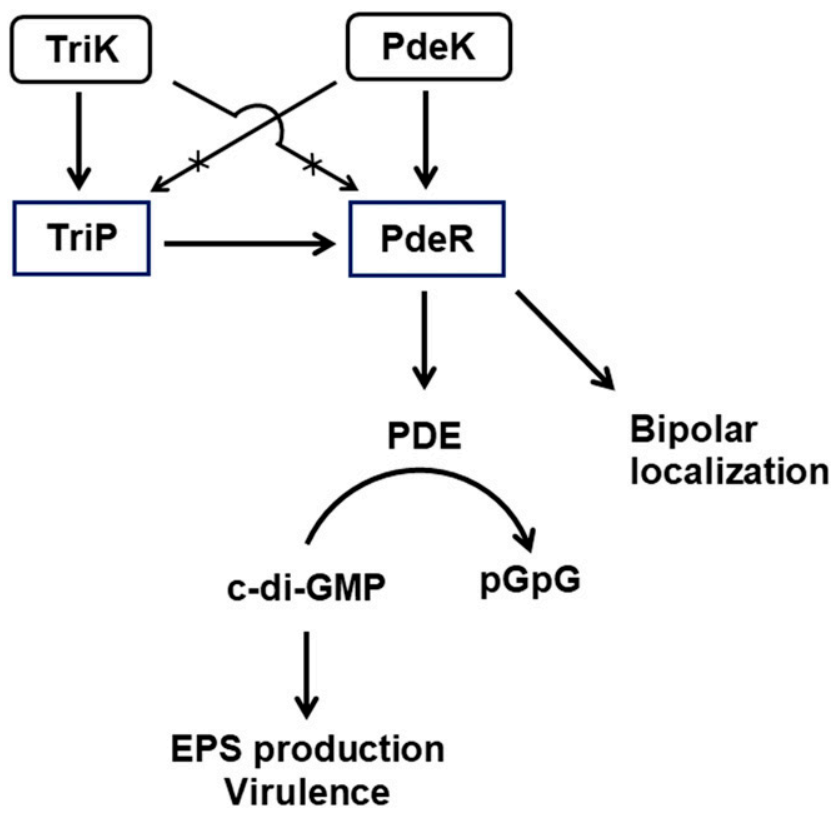

Fig. 8. A proposal model outlining TriP regulation of virulence expression via interacting with PdeR in Xanthomonas oryzae pv. oryzae. The response regulator PdeR has been shown to function as a phosphodiesterase (PDE) for cyclic dimeric guanosine monophosphate (c-di-GMP) hydrolysis and form a two-component system with the histidine kinase PdeK (Yang et al. 2012). TriP, the putative response regulator with the REC and helixturn-helix domains specifically interact with the PdeR but not PdeK. The REC domain of TriP and the EAL domain of PdeR are essential for the proteinprotein interaction. Such an interaction significantly impacts PdeR bipolar localization and PDE ability to degrade c-di-GMP into pGpG, thereby regulating bacterial virulence and exopolysaccharides (EPS) production in a c-di-GMP signaling-dependent manner. 
For purification of the GST-fusion proteins, the following protocol was used. In short, expression of the target protein was induced by the addition of isopropyl- $\beta$-D-thiogalactoside at a final concentration of $0.1 \mathrm{mM}$ in the bacterial culture M9. The cells were chilled to $4^{\circ} \mathrm{C}$ and were collected by centrifugation. The cell pellets were resuspended in $10 \mathrm{mM}$ Tris- $\mathrm{HCl}(\mathrm{pH} 8.0)$, followed by sonication. The soluble protein fractions were collected by centrifugation and were mixed with pre-equilibrated glutathione sepharose 4B (GE Healthcare, Piscataway, NJ, U.S.A.). The proteins were washed with $1 \times$ phosphate buffered saline (PBS) ( $\mathrm{pH} 7.3)$ and were eluted with an elution buffer
(pH 8.0). pET28a-His ${ }_{6}$-fusion proteins were purified through a Ni-binding column. The soluble protein fractions were mixed with pre-equilibrated $\mathrm{Ni}_{2}$ resin (GE Healthcare) and were eluted with a buffer containing $300 \mathrm{mM}$ imidazole. The GST tag expressed from pGEX-6P-1 was purified using the same procedure as a negative control. Protein concentration was assayed using the Bradford method (CWBIO, Beijing).

\section{GST pull-down assay.}

Pull-down experiments were performed with purified recombinant proteins. In short, $0.1 \mathrm{mg}$ of GST-tagged recombinant proteins

Table 1. Bacterial strains and plasmids used in this study

\begin{tabular}{|c|c|c|}
\hline Strains or plasmids & Relevant characteristics ${ }^{a}$ & Reference or source \\
\hline \multicolumn{3}{|l|}{ Escherichia coli } \\
\hline DH5 $\alpha$ & 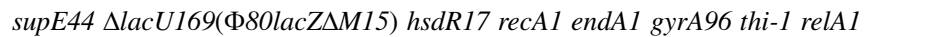 & Hanahan 1983 \\
\hline BL21 & For protein expression & Tiangen Biotech Co. \\
\hline \multicolumn{3}{|c|}{ Xanthomonas oryzae pv. oryzae } \\
\hline PXO99 & Wild-type strain, Philippine race $6, \mathrm{Cp}^{\mathrm{r}}$ & Lab collection \\
\hline$\Delta p d e R$ & pdeR gene-deletion mutant derived from $\mathrm{PXO} 99^{\mathrm{A}}, \mathrm{Gm}^{\mathrm{r}}, \mathrm{Cp}^{\mathrm{r}}$ & Yang et al. 2012 \\
\hline$\Delta$ triP & triP gene-deletion mutant derived from PXO99 ${ }^{\mathrm{A}}, \mathrm{Cp}^{\mathrm{r}}$ & This study \\
\hline$\Delta$ triP(pBTriP) & $\Delta$ triP containing plasmid $\mathrm{pBTriP}, \mathrm{Ap}^{\mathrm{r}}, \mathrm{Cp}^{\mathrm{r}}$ & This study \\
\hline$\Delta$ triPApdeR & triP and pdeR double-deletion mutant. $\mathrm{Cp}^{\mathrm{r}}$ & This study \\
\hline$\Delta$ triP(pBPdeR-GFP) & $\Delta$ triP containing plasmid $\mathrm{pBPdeR}-\mathrm{GFP}, \mathrm{Ap}^{\mathrm{r}}, \mathrm{Cp}^{\mathrm{r}}$ & This study \\
\hline$\Delta p d e R(\mathrm{pBTriP})$ & $\Delta p d e R$ containing plasmid $\mathrm{pBTriP}, \mathrm{Ap}^{\mathrm{r}}, \mathrm{Cp}^{\mathrm{r}}$ & This study \\
\hline \multicolumn{3}{|l|}{ Saccharomyces cerevisiae } \\
\hline Y2HGold & 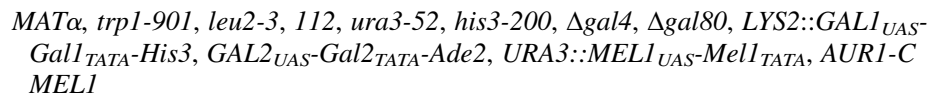 & Clontech \\
\hline Y187 & 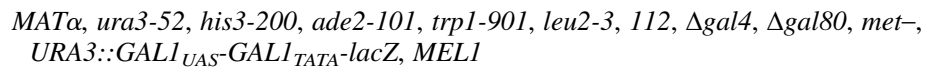 & Clontech \\
\hline \multicolumn{3}{|c|}{ - } \\
\hline pMD18-T & Cloning vector, $\mathrm{Ap}^{\mathrm{r}}$ & TaKaRa, Tokyo \\
\hline pET28a & Expression vector to generate a $\mathrm{N}$-terminal $\mathrm{His}_{6}$-tag, $\mathrm{Km}^{\mathrm{r}}$ & $\begin{array}{l}\text { Novagen, Madison, } \\
\text { WI, U.S.A. }\end{array}$ \\
\hline pGEX-6P-1 & Expression vector to generate a N-terminal GST-tag, Ap ${ }^{r}$ & Lab collection \\
\hline pKMS1 & Suicidal vector carrying $s a c B$ gene for nonmarker mutagenesis, $\mathrm{Km}^{\mathrm{r}}$ & Li et al. 2011 \\
\hline pBBR1MCS-4 & Broad-host range expression vector, $\mathrm{Ap}^{\mathrm{r}}$ & Kovach et al 1995 \\
\hline pGADT7 & $\begin{array}{l}\text { Yeast two-hybrid vector to create fusion protein containing the GAL4 activation } \\
\text { domain, } \mathrm{Ap}^{\mathrm{r}}\end{array}$ & Clontech \\
\hline pGBKT7 & $\begin{array}{l}\text { Yeast two-hybrid vector to create fusion protein containing GAL4 DNA binding } \\
\text { domain, } \mathrm{Km}^{\mathrm{r}}\end{array}$ & Clontech \\
\hline pGBKPdeR & pGBKT7 carrying full-length $p d e R, \mathrm{Km}^{\mathrm{r}}$ & Yang et al. 2012 \\
\hline pETPdeR & pET28a carrying full-length $p d e R, \mathrm{Km}^{\mathrm{r}}$ & Yang et al. 2012 \\
\hline pETPdeK & pET28a carrying full-length $p d e K, \mathrm{Km}^{\mathrm{r}}$ & Yang et al. 2012 \\
\hline pMDTriP & pMD18-T derivative carrying full-length triP, $\mathrm{Ap}^{\mathrm{r}}$ & This study \\
\hline pMDPdeR & pMD18-T derivative carrying full-length $p d e R, \mathrm{Ap}^{\mathrm{r}}$ & This study \\
\hline pMDTriP-N & pMD18-T derivative carrying the REC domain of triP, $\mathrm{Ap}^{\mathrm{r}}$ & This study \\
\hline pMDTriP-C & pMD18-T derivative carrying the HTH domain of triP, $\mathrm{Ap}^{\mathrm{r}}$ & This study \\
\hline pMDPdeR-G & pMD18-T derivative carrying the GGDEF domain of $p d e R, \mathrm{Ap}^{\mathrm{r}}$ & This study \\
\hline pMDPdeR-E & pMD18-T derivative carrying the EAL domain of $p d e R, \mathrm{Ap}^{\mathrm{r}}$ & This study \\
\hline pMDPdeR-R & pMD18-T derivative carrying the REC domain of $p d e R, \mathrm{Ap}^{\mathrm{r}}$ & This study \\
\hline pMDGFP & pMD18-T derivative carrying full-length $G F P, \mathrm{Ap}^{\mathrm{r}}$ & This study \\
\hline pMDPdeR-GFP & pMD18-T derivative carrying full-length $p d e R$ (without stop codon), $\mathrm{Ap}^{\mathrm{r}}$ & This study \\
\hline pMDTriP-GFP & pMD18-T derivative carrying full-length triP (without stop codon), $\mathrm{Ap}^{\mathrm{r}}$ & This study \\
\hline pMDPdeK-GFP & pMD18-T derivative carrying full-length $p d e K$ (without stop codon), Apr & This study \\
\hline pGADTriP & pGADT7 carrying full-length triP, $\mathrm{Ap}^{\mathrm{r}}$ & This study \\
\hline pGADTriP-C & pGADT7 carrying the DNA fragment of triP HTH domain, Ap ${ }^{r}$ & This study \\
\hline pGADTriP-N & pGADT7 carrying the DNA fragment of triP REC domain, $\mathrm{Ap}^{\mathrm{r}}$ & This study \\
\hline pGADPdeR-E & pGADT7 carrying the DNA fragment of $p d e R$ EAL domain, Ap ${ }^{r}$ & This study \\
\hline pGBKPdeR-E & pGBKT7 carrying the DNA fragment of pdeR EAL domain, $\mathrm{Km}^{\mathrm{r}}$ & This study \\
\hline pGBKPdeR-G & pGBKT7 carrying the DNA fragment of $p d e R$ GGDEF domain, $\mathrm{Km}^{\mathrm{r}}$ & This study \\
\hline pGBKPdeR-R & pGBKT7 carrying the DNA fragment of $p d e R$ REC domain, $\mathrm{Km}^{\mathrm{r}}$ & This study \\
\hline pGBKTriP-N & pGBKT7 carrying the DNA fragment of triP REC domain, $\mathrm{Km}^{\mathrm{r}}$ & This study \\
\hline pGEXTriP & pGEX-6P-1 carrying full-length triP, $\mathrm{Ap}^{\mathrm{r}}$ & This study \\
\hline pKMTriP & pKMS1 derivative carrying a triP mutation, $\mathrm{Km}^{\mathrm{r}}$ & This study \\
\hline pKMPdeR & pKMS1 derivative carrying a pde $R$ mutation, $\mathrm{Km}^{\mathrm{r}}$ & This study \\
\hline pBTriP & pBBR1MCS-4 carrying the full-length of triP, Ap ${ }^{\mathrm{r}}$ & This study \\
\hline pBGFP & GFP fragment ligated into vector pBBR1MCS-4, Ap ${ }^{r}$ & This study \\
\hline pBPdeR-GFP & pBGFP carrying full-length $p d e R, \mathrm{Ap}^{\mathrm{r}}$ & This study \\
\hline pBTriP-GFP & pBGFP carrying full-length triP, $\mathrm{Ap}^{\mathrm{r}}$ & This study \\
\hline pBPdeK-GFP & pBGFP carrying full-length $p d e K, \mathrm{Ap}^{\mathrm{r}}$ & This study \\
\hline
\end{tabular}

\footnotetext{
${ }^{\mathrm{a}} \mathrm{Ap}^{\mathrm{r}}, \mathrm{Cp}^{\mathrm{r}}, \mathrm{Gm}^{\mathrm{r}}$, and $\mathrm{Km}^{\mathrm{r}}$ indicate resistance to antibiotics ampicillin, cephalexin, gentamycin, and kanamycin, respectively. $\mathrm{HTH}=$ helix-turn-helix.
} 
were mixed with $0.1 \mathrm{mg}$ of $\mathrm{His}_{6}$-tagged proteins and were adjusted to a 1-ml volume using PBS. After being thoroughly mixed at room temperature, the glutathione sepharose $4 \mathrm{~B}$ beads $\left(200 \mu \mathrm{l}\right.$ slurry) were added and incubated at $4{ }^{\circ} \mathrm{C}$ overnight. Coeluted proteins were separated by sodium dodecyl sulfatepolyacrylamide gel electrophoresis and were analyzed by immunoblotting using anti-GST and anti-His monoclonal antibodies (Beijing Protein Innovation, Beijing). Primary antibodies were recognized by goat antimouse secondary antibodies conjugated with horseradish peroxidase, which were directly visualized by applying the TMB substrate (Promega, Beijing). The purified GST protein was incubated with the $\mathrm{His}_{6}$-tagged protein and was subjected to the same procedures to serve as a negative control.

\section{PDE colorimetric assay.}

The PDE activity was tested as previously described (Bobrov et al. 2005; Yang et al. 2012). In brief, $5 \mathrm{mM}$ bis (p-nitrophenyl) phosphate substrate and $20 \mu \mathrm{g}$ of purified proteins were incubated at $37^{\circ} \mathrm{C}$ for $1.5 \mathrm{~h}$ in reaction buffer $(50 \mathrm{mM}$ Tris- $\mathrm{HCl}$, $1 \mathrm{mM} \mathrm{MnCl}_{2}, \mathrm{pH} 8.5$ ). The hydrolysis of substrate was detected at $410 \mathrm{~nm}$ by spectrophotometer. To test the effect of TriP on PDE activity of PdeR, $200 \mu \mathrm{M}$ ATP and $20 \mu \mathrm{g}$ of PdeK were incubated at $25^{\circ} \mathrm{C}$ for $3 \mathrm{~min}$ in phosphorylation buffer $(25 \mathrm{mM}$ Tris- $\mathrm{HCl}, 50 \mathrm{mM} \mathrm{NaCl}, 5 \mathrm{mM} \mathrm{MgCl}_{2}, 1 \mathrm{mM}$ dithiothreitol, $0.5 \mathrm{mM}$ EDTA, pH 8.0) to a volume of $100 \mu \mathrm{l}$. Then, $20 \mu \mathrm{g}$ of purified PdeR protein and $20 \mu \mathrm{g}$ of purified TriP protein were added and incubated at $25^{\circ} \mathrm{C}$ for $20 \mathrm{~min}$. Finally, $5 \mathrm{mM}$ bis (pnitrophenyl) phosphate substrate were added for the PDE colorimetric reaction and were incubated at $37^{\circ} \mathrm{C}$ for $2 \mathrm{~h}$; the final volume was $200 \mu \mathrm{l}$. The hydrolysis of substrate was detected at $410 \mathrm{~nm}$ by spectrophotometer. Bovine serum albumin was used as a negative control. The experiment was repeated three times with three technical replicates.

\section{Gene deletion mutagenesis and complementation.}

The mutant was generated using homologous recombination by the suicide vector pKMS1 ( $\mathrm{Li}$ et al. 2011). The DNA fragments corresponding to downstream and upstream regions of triP and $p d e R$ were amplified, using primer pairs KMTriPLF/R, KMTriPRF/R, KMPdeRLF/R, and KMPdeRRF/R, respectively, and were cloned to the middle vector pMD18-T for sequencing. The pMD18-T-derived constructs were digested with corresponding restriction enzymes and were ligated into the pKMS1 suicide vector, resulting in plasmids pKMTriP and pKMPdeR. For the $\Delta$ triP deletion mutant, the plasmid pKMTriP was introduced into PXO99 ${ }^{\mathrm{A}}$ by electroporation, while for the $\triangle t r i P \Delta p d e R$ double mutant, the plasmid pKMPdeR was introduced into the triP mutant. Potential mutants were selected on NAN medium ( $1 \%$ tryptone, $0.1 \%$ yeast extract, $0.3 \%$ peptone, $1.5 \%$ agar). The single colonies were inoculated into NBN broth (1\% tryptone, $0.1 \%$ yeast extract, $0.3 \%$ peptone) for selection against the $s a c B$ gene marker. Bacterial cells were diluted to appropriate concentrations and were plated on NAS (1\% tryptone, $0.1 \%$ yeast extract, $10 \%$ sucrose, $0.3 \%$ peptone, $1.5 \%$ agar) for selection of mutants. The mutant candidates that can grow on NAS but are sensitive to kanamycin were further confirmed by PCR. For complementation analysis, the coding region for full-length triP was amplified by primer pair BTriPF/R, ligated into vector pBBR1MCS-4 and was electroporated into the $\Delta$ triP mutant for complementation studies.

\section{Determination of the intracellular c-di-GMP concentration.}

Intracellular c-di-GMP concentrations were determined by LC-MS/MS, using a method that has been previously described
(Yuan et al. 2015). In brief, bacterial cells were cultured at $28^{\circ} \mathrm{C}$ in M210 overnight and were then subcultured into $100 \mathrm{ml}$ of fresh media (1:100 dilution). The cultures were grown to mid-to-late $\log$ phase $\left(\mathrm{OD}_{600}=2.5\right)$. Cells were collected by centrifugation at $9,000 \times g$ for $20 \mathrm{~min}$ and were then resuspend in $1 \mathrm{ml}$ of cold extraction buffer by pipetting (40\% methanol and $40 \%$ acetonitrile in $0.1 \mathrm{~N}$ formic acid). The mixture was incubated at $-20^{\circ} \mathrm{C}$ overnight, before ultrasonication treatment. The supernatant was collected after centrifugation at max speed for 10 min twice. Supernatant of each sample $(100 \mu \mathrm{l})$ was analyzed by LCMS/MS. The experiment was independently repeated three times.

\section{Subcellular protein localization.}

The full length of the $g f p$ gene was amplified by PCR using primer pair BGFPF/R and was ligated into a broad host-range vector $\mathrm{pBBR} 1 \mathrm{MCS}-4$, resulting in $\mathrm{pBGFP}$. We used the primer pairs BpdeR-GFPF/R, BpdeK-GFPF/R, and BTriP-GFPF/R to clone $p d e R$, pdeK, and triP. The fragments were then subcloned into pBGFP, resulting in pBPdeR-GFP, pBPdeK-GFP, and pBTriP-GFP. The constructs were transformed into different PXO99 ${ }^{\mathrm{A}}$ strains. Expressions of fusion proteins were confirmed by Western blotting, using anti-GFP primary antibodies (Abmart, Shanghai, China). Goat antimouse immunoglobulin G horseradish peroxidase conjugate secondary antibody (Abmart) was applied to recognize the primary antibodies.

Samples were prepared for microscopy as previously described (Xue et al. 2018). Different strains were grown in M210 to an $\mathrm{OD}_{600}$ of 0.8 . Bacterial cells were diluted, $3 \mu \mathrm{l}$ of cell suspension was spotted on a glass slide that was freshly coated with $1.0 \%$ agarose, and the suspension was covered with a coverslip. Fluorescence images were acquired using an Olympus BX61 microscope with GFP and fluorescein isothiocyanate filters and were recorded with a cooled CCD camera DP72 (Olympus, Tokyo) at subsaturating emission intensities. The subcellular localization of cells was determined as described previously (Shikuma et al. 2012). At least 10 fields of view, each containing more than 100 cells were observed. The experiment was independently repeated three times.

\section{Virulence assays.}

The susceptible rice variety Oryza sativa L. cv. Nipponbare was used to test the virulence of $X$. oryzae pv. oryzae strains. Virulence assays were performed on 6-week-old rice plants using the leaf-clip inoculation method (Ray et al. 2000). Different PXO99 ${ }^{\mathrm{A}}$ strains were incubated at $28^{\circ} \mathrm{C}$ with agitation (approximately $200 \mathrm{rpm}$ ) until they reached an $\mathrm{OD}_{600}=0.8$ to $1.0 \mathrm{in}$ the M210 medium with relevant antibiotics. The cells were collected by centrifugation and were resuspended in sterilized distilled water. The concentration of this suspension was adjusted to an $\mathrm{OD}_{600}=0.8$. Symptoms were photographed and lesion lengths were measured for individual inoculated leaves 14 days after inoculation. Twenty leaves were inoculated for each strain. The experiment was independently repeated four times.

\section{Bacterial growth curve assays.}

The bacterial growth assay was performed as previously described (Tian et al. 2015). In brief, X. oryzae pv. oryzae wild type and mutant strains were grown in M210 liquid medium overnight at $28^{\circ} \mathrm{C}$; then, these strains were diluted in $\mathrm{M} 210$ medium to a final cell density $\left(\mathrm{OD}_{600}=0.01\right)$. The diluted cells were cultured at $28^{\circ} \mathrm{C}$ with $200 \mathrm{rpm}$, and the bacterial population was measured after every $12 \mathrm{~h}$. The experiment was independently repeated twice.

\section{EPS production assays.}

EPS production was assayed as previously described (Xue et al. 2018). The bacterial culture $\left(\mathrm{OD}_{600}=2.5\right)$ were centrifuged 
at $8,000 \mathrm{rpm}$ for $20 \mathrm{~min}$. Then, the supernatant was harvested, two volumes of absolute ethanol were added, and the mixture was kept at $-20^{\circ} \mathrm{C}$ overnight. The precipitated EPS was collected by centrifugation and was dried before determination of dry weight. For the plate assays, bacterial strains were grown in M210 until they reached an $\mathrm{OD}_{600}=0.8$, and $2 \mu \mathrm{l}$ of bacterial culture was spotted onto a PSA plate without any antibiotic and was incubated at $28^{\circ} \mathrm{C}$ for 4 days before photos were taken for the colonies. The experiment was repeated three times.

\section{Statistical analysis.}

All analysis was performed using SPSS 14.0 (SPSS Inc., Chicago) and Fisher's least significant difference test was used to determine significant differences in the assays for PDE activity, disease lesion length, in vivo growth, and EPS production.

\section{ACKNOWLEDGMENTS}

H. Li, and D. Xue performed experiments; H. Li, D. Xue, F. Tian, X. Yuan, F. Yang, H. Chen, C. Yang, and C. He analyzed data; H. Li, F. Tian, W. Hutchins, C. Yang, and C. He wrote the manuscript.

\section{LITERATURE CITED}

Abel, S., Chien, P., Wassmann, P., Schirmer, T., Kaever, V., Laub, M. T., Baker, T. A., and Jenal, U. 2011. Regulatory cohesion of cell cycle and cell differentiation through interlinked phosphorylation and second messenger networks. Mol. Cell 43:550-560.

Bobrov, A. G., Kirillina, O., and Perry, R. D. 2005. The phosphodiesterase activity of the HmsP EAL domain is required for negative regulation of biofilm formation in Yersinia pestis. FEMS Microbiol. Lett. 247: 123-130.

Bourret, R. B. 2010. Receiver domain structure and function in response regulator proteins. Curr. Opin. Microbiol. 13:142-149.

Dahlstrom, K. M., Giglio, K. M., Collins, A. J., Sondermann, H., and O'Toole, G. A. 2015. Contribution of physical interactions to signaling specificity between a diguanylate cyclase and its effector. MBio 6 : e01978-15.

Fang, X., and Gomelsky, M. 2010. A post-translational, c-di-GMPdependent mechanism regulating flagellar motility. Mol. Microbiol. 76: 1295-1305.

Gao, R., and Stock, A. M. 2009. Biological insights from structures of two-component proteins. Annu. Rev. Microbiol. 63:133-154.

Güvener, Z. T., and Harwood, C. S. 2007. Subcellular location characteristics of the Pseudomonas aeruginosa GGDEF protein, WspR, indicate that it produces cyclic-di-GMP in response to growth on surfaces. Mol. Microbiol. 66:1459-1473.

Hanahan, D. 1983. Studies on transformation of Escherichia coli with plasmids. J. Mol. Biol. 166:557-580.

Hengge, R. 2016. Trigger phosphodiesterases as a novel class of c-di-GMP effector proteins. Philos. Trans. R. Soc. Lond. B Biol. Sci. 371: 20150498.

Hopkins, C. M., White, F. F., Choi, S. H., Guo, A., and Leach, J. E. 1992. Identification of a family of avirulence genes from Xanthomonas oryzae pv. oryzae. Mol. Plant-Microbe Interact. 5:451-459.

Jones, C. J., Utada, A., Davis, K. R., Thongsomboon, W., Zamorano Sanchez, D., Banakar, V., Cegelski, L., Wong, G. C. L., and Yildiz, F. H. 2015. C-di-GMP regulates motile to sessile transition by modulating MshA pili biogenesis and near-surface motility behavior in Vibrio cholerae. PLoS Pathog. 11:e1005068.

Jung, K., Fried, L., Behr, S., and Heermann, R. 2012. Histidine kinases and response regulators in networks. Curr. Opin. Microbiol. 15:118-124.

Kovach, M. E., Elzer, P. H., Hill, D. S., Robertson, G. T., Farris, M. A., Roop, R. M., 2nd, and Peterson, K. M. 1995. Four new derivatives of the broad-host-range cloning vector $\mathrm{pBBR} 1 \mathrm{MCS}$, carrying different antibioticresistance cassettes. Gene 166:175-176.

Li, B., Tian, F., Chen, H., Wu, M., and He, C. 2013. [Binding of transcription regulator Clpxoo to promoter of endoglucanase gene engAxoo was inhibited by c-di-GMP in Xanthomonas oryzae pv. oryzae.]. Wei Sheng Wu Xue Bao 53:1166-1171.

Li, Y. R., Zou, H. S., Che, Y. Z., Cui, Y. P., Guo, W., Zou, L. F., Chatterjee, S., Biddle, E. M., Yang, C. H., and Chen, G. Y. 2011. A novel regulatory role of HrpD6 in regulating hrp-hrc-hpa genes in Xanthomonas oryzae pv. oryzicola. Mol. Plant-Microbe Interact. 24:1086-1101.
Lindenberg, S., Klauck, G., Pesavento, C., Klauck, E., and Hengge, R. 2013. The EAL domain protein YciR acts as a trigger enzyme in a c-diGMP signalling cascade in E. coli biofilm control. EMBO J. 32: 2001-2014.

Merritt, J. H., Ha, D. G., Cowles, K. N., Lu, W., Morales, D. K., Rabinowitz, J., Gitai, Z., and O'Toole, G. A. 2010. Specific control of Pseudomonas aeruginosa surface-associated behaviors by two c-di-GMP diguanylate cyclases. MBio 1:e0183-10.

Niño-Liu, D. O., Ronald, P. C., and Bogdanove, A. J. 2006. Xanthomonas oryzae pathovars: Model pathogens of a model crop. Mol. Plant Pathol. 7:303-324.

Paul, K., Nieto, V., Carlquist, W. C., Blair, D. F., and Harshey, R. M. 2010. The c-di-GMP binding protein YcgR controls flagellar motor direction and speed to affect chemotaxis by a "backstop brake" mechanism. Mol. Cell 38:128-139.

Paul, R., Jaeger, T., Abel, S., Wiederkehr, I., Folcher, M., Biondi, E. G., Laub, M. T., and Jenal, U. 2008. Allosteric regulation of histidine kinases by their cognate response regulator determines cell fate. Cell 133:452-461.

Povolotsky, T. L., and Hengge, R. 2012. 'Life-style' control networks in Escherichia coli: Signaling by the second messenger c-di-GMP J. Biotechnol. 160:10-16.

Ray, S. K., Rajeshwari, R., and Sonti, R. V. 2000. Mutants of Xanthomonas oryzae pv. oryzae deficient in general secretory pathway are virulence deficient and unable to secrete xylanase. Mol. Plant-Microbe Interact. 13:394-401.

Roelofs, K. G., Jones, C. J., Helman, S. R., Shang, X., Orr, M. W., Goodson, J. R., Galperin, M. Y., Yildiz, F. H., and Lee, V. T. 2015. Systematic Identification of cyclic-di-GMP binding proteins in Vibrio cholerae reveals a novel class of cyclic-di-GMP-binding ATPases associated with type II secretion systems. PLoS Pathog. 11:e1005232.

Römling, U., Galperin, M. Y., and Gomelsky, M. 2013. Cyclic di-GMP The first 25 years of a universal bacterial second messenger. Microbiol. Mol. Biol. Rev. 77:1-52.

Römling, U., Liang Z.-X., and Dow, J. M. 2017. Progress in understanding the molecular basis underlying functional diversification of cyclic dinucleotide turnover proteins. J. Bacteriol. 199:e00790-16.

Schirmer, T., and Jenal, U. 2009. Structural and mechanistic determinants of c-di-GMP signalling. Nat. Rev. Microbiol. 7:724-735.

Shikuma, N. J., Fong, J. C., and Yildiz, F. H. 2012. Cellular levels and binding of c-di-GMP control subcellular localization and activity of the Vibrio cholerae transcriptional regulator VpsT. PLoS Pathog. 8: e1002719.

Simm, R., Morr, M., Kader, A., Nimtz, M., and Römling, U. 2004. GGDEF and EAL domains inversely regulate cyclic di-GMP levels and transition from sessility to motility. Mol. Microbiol. 53:1123-1134.

Skerker, J. M., Prasol, M. S., Perchuk, B. S., Biondi, E. G., and Laub, M. T. 2005. Two-component signal transduction pathways regulating growth and cell cycle progression in a bacterium: A system-level analysis. PLoS Biol. 3:e334.

Sondermann, H., Shikuma, N. J., and Yildiz, F. H. 2012. You've come a long way: c-di-GMP signaling. Curr. Opin. Microbiol. 15:140-146.

Stock, A. M., Robinson, V. L., and Goudreau, P. N. 2000. Two-component signal transduction. Annu. Rev. Biochem. 69:183-215.

Sun, Q. H., Hu, J., Huang, G. X., Ge, C., Fang, R. X., and He, C. Z. 2005. Type-II secretion pathway structural gene xpsE, xylanase- and cellulase secretion and virulence in Xanthomonas oryzae pv. oryzae. Plant Pathol. 54:15-21.

Tian, F., Yu, C., Li, H., Wu, X., Li, B., Chen, H., Wu, M., and He, C. 2015. Alternative sigma factor RpoN2 is required for flagellar motility and full virulence of Xanthomonas oryzae pv. oryzae. Microbiol. Res. 170:177-183.

White, F. F., and Yang, B. 2009. Host and pathogen factors controlling the rice-Xanthomonas oryzae interaction. Plant Physiol. 150:1677-1686.

Xue, D. R., Tian, F., Yang, F. H., Chen, H. M., Yuan, X. C., Yang, C.-H., Chen, Y. H., Wang, Q., and He, C. Y. 2018. Phosphodiesterase EdpX1 promotes Xanthomonas oryzae pv. oryzae virulence, exopolysaccharide production, and biofilm formation. Appl. Environ. Microbiol. 84 e01717-e01718.

Yang, F., Qian, S., Tian, F., Chen, H., Hutchins, W., Yang, C.-H., and He, C. 2016. The GGDEF-domain protein GdpX1 attenuates motility, exopolysaccharide production and virulence in Xanthomonas oryzae pv. oryzae. J. Appl. Microbiol. 120:1646-1657.

Yang, F., Tian, F., Chen, H., Hutchins, W., Yang, C.-H., and He, C. 2015. The Xanthomonas oryzae pv. oryzae PilZ-domain proteins function differentially in cyclic di-GMP binding and regulation of virulence and motility. Appl. Environ. Microbiol. 81:4358-4367.

Yang, F., Tian, F., Li, X., Fan, S., Chen, H., Wu, M., Yang, C.-H., and He, C. 2014. The degenerate EAL-GGDEF domain protein Filp functions as a 
cyclic di-GMP receptor and specifically interacts with the PilZ-domain protein PXO_02715 to regulate virulence in Xanthomonas oryzae pv. oryzae. Mol. Plant-Microbe Interact. 27:578-589.

Yang, F., Tian, F., Sun, L., Chen, H., Wu, M., Yang, C.-H., and He, C. 2012. A novel two-component system PdeK/PdeR regulates c-di-GMP turnover and virulence of Xanthomonas oryzae pv. oryzae. Mol. PlantMicrobe Interact. 25:1361-1369.

Yuan, X., Khokhani, D., Wu, X., Yang, F., Biener, G., Koestler, B. J., Raicu, V., He, C., Waters, C. M., Sundin, G. W., Tian, F., and Yang, C.-H. 2015. Cross-talk between a regulatory small RNA, cyclic-di-GMP signalling and flagellar regulator FlhDC for virulence and bacterial behaviours. Environ. Microbiol. 17:4745-4763.

Zhang, H., and Wang, S. 2013. Rice versus Xanthomonas oryzae pv. oryzae: A unique pathosystem. Curr. Opin. Plant Biol. 16:188-195.
Zhou, L., Huang, T. W., Wang, J. Y., Sun, S., Chen, G., Poplawsky, A., and He, Y. W. 2013. The rice bacterial pathogen Xanthomonas oryzae pv. oryzae produces 3-hydroxybenzoic acid and 4-hydroxybenzoic acid via XanB2 for use in xanthomonadin, ubiquinone, and exopolysaccharide biosynthesis. Mol. Plant-Microbe Interact. 26: 1239-1248.

Zschiedrich, C. P., Keidel, V., and Szurmant, H. 2016. Molecular mechanisms of two-component signal transduction. J. Mol. Biol. 428: 3752-3775.

\section{AUTHOR-RECOMMENDED INTERNET RESOURCE}

BLASTn search tool: https://blast.ncbi.nlm.nih.gov/Blast.cgi 